\section{A CASE OF PNEUMOTHORAX.1}

BY J. F. A. ADAMS, M.D., PIT'RSFIELD, MASS.

THE interesting features of this case are that it was a simple pneumothorax, of peculiar origin, persisting for five years and terminating in spontaneous recovery.

A man, aged forty-six, of American birth and parentage, widower, carriage-painter by trade, was admitted to the Pittsfield House of Mercy as a patient, February 3d, 1881. The previous history of the case, as learned from his own statements, is as follows : 'Two years previously, having, up to that time enjoyed perfect health, he took a severe cold and was attacked with what was called by his physician "pleuro-pneumouia" of the left side. After being ill, as he thinks, about three days, while straining at stool, he was suddenly attacked with dyspnœa, which had continued without relief up to the time of admission. 'Two or. three days after the dyspnoea commenced, he began to cough and had coughed ever since, usually without expectoration. He had lost appetite, flesh and strength, but had not been confined to bed. A few weeks before admission he was examined by Dr. F. K. Paddock, who found tympanitic resonance and absence of respiratory sounds on the left side. He then consulted Dr. Austin Flint, Sr., who, in a letter to Dr. Paddock, gave a guarded diagnosis, but suggested that the obstructed respiration might be due to pressure upon the left primary bronchus by an aneurism of the aorta; though no physical signs of aneurism were discoverable, and this diagnosis was made only by exclusion.

On admission, he came under the care of Dr. Vermilye, who found him feeble and haggard, with a small and compressible pulse of 86 . No pain in the chest, but considerable dyspnoea, especially on exertion. Left chest tympanitic on percussion, with an entire absence of respiratory murmur. He was treated with potassium iodide in five-grain doses, with quinine and iron.

A month later, a faint vesicular murmur could be heard over a limited area of the upper portion and front of left chest, and pain was felt in the same region. Otherwise his condition was unchanged. The iodine was continued till June 1st, a period of four months, after which he took grindelia robusta for two months, neither remedy producing any apparent change in his condition.

On October 6th, eight months after admission, he was examined by myself, and the following record made :-

Left semi-circumference of chest 17 1-2 inches.

Right semi-circumference of chest $161-2$ inches.

Extreme expansion 3-4 inch.

Expansion of left side 1-4 inch.

Expansion of right side 1-2 inch.

Intercostal spaces fuller on left side than right, and palpation shows left side nearly immobile during respiration. Left side of chest everywhere tympanitic, about equally so over all portions. The tympanitic resonance in firont extends to the right margin of the sternum. Cardiac dulness entirely to right of sternum, extending from its right margin to a line one inch to right of the right nipple, and from the third to the sixth rib. Apex-beat close to the right edge of sternum at level of sixth rib. Vesicular murmur heard

1 Read before the Berkshire District Medical Society, September very faintly over a very small area on left side, at the level of axilla, both in front and behind. Elsewhere, on the left side no sound whatever can be detected. On the right side, respiration is normal. No aneurism or nurmur can be detected. Patient still suffers with dyspnoa, which is constant, even when at rest, and excessive on exertion. He complains of a feeling of weight or oppression in the chest, especially at the upper part, amounting sometimes to pain. Pulse 80 , soft and weak. Is still feeble and has not materially changed since admission. Has an occasional dry cough.

This patient became a permanent resident at the House of Mercy, remaining there for nearly four years. For three years there was but little change in his condition. He was examined from time to time by myself, as well as by several others of the attending physicians, and the physical sigus were found unchanged. He was debilitated, but not confined to bed, went out in good weather and spent his time in reading and acquiring the art of stenography. For treatment he had only tonics and general good care. He left the Hospital for six weeks in February and March, 1883, thinking himself able to go to work, but returned with a severe attack of bronchitis on the right side. This soon passed off, and no change took place until about March Ist, 1884.

At this time he noticed that he could feel the heart beating at a point a little to the left of the position it had so long occupied; and thereafter he was conscious of a gradual progress of the apex-beat toward the left, until, by the latter part of May, he could feel it in its normal position, the change laving taken place in something less than three months. As the heart moved, the dyspnœa gradually diminished, and he soon began to regain flesh and strength. I was away at the time the cliange occurred, and did not examine him until the 25th of the following September, when his condition was as follows :-

Resonance on percussion normal and equal over both sides of chest. Vesicular murmur is now heard in its normal situation, over both sides, the only difference being that it is not quite so loud and strong on the left, where it has also a slight tremulousness or cog-wheel character. Apex-beat of heart is found one and one-half inches below and one inch to the left of the left nipple, being six inches in a direct line from its former position. Area of cardiac dulness somewhat greater than normal. He is now able to walk at a brisk pace without dyspncea.

He remained at the House of Mercy till January 1, 1885, when he was discharged well. Since then he has enjoyed good health, and has been able to earn his living as a stenographer. His normal weight before this illness was one hundred and forty-five to one hundred and sixty pounds. While at the House of Mercy he weighed one hundred and twenty-five pounds, increasing to one hundred and forty before his discharge. His present weight is one hundred and eighty. He writes me that he has no cough, dyspnœa, nor pain in the chest. He still feels the apex-beat to the left of the nipple.

The diagnosis of pneumothorax in this case was based upon the following symptoms :

First. Uniform tympanitic resonance over the left chest.

Second. Distension of the affected side. 29 th, 1886. 
Fourth. Displacement of the heart.

The only question could be between this and a unilateral, pulmonary emphysema, but in emphysema we never get the absolute stillness which characterized the affected side in this case, but have, on the contrary, a prolonged and sibilant expiration. In emphysema, also, the tympanitic resonance varies in intensity over different portions of the chest; displacement of the heart, if it occurs, is not excessive, and the character of the dyspnoa is not the same, being distinguished by the long and laborious expiration. In this case the dyspnoea was simply a shortness of the breath, without laborious expiration; it was constant, and aggravated by exertion, while in emphysema there is a paroxysmal tendency.

In seeking for the cause of this attack, we have for a guide only the patient's statement that the dyspncea came on suddenly, while straining at stool, after suffering for three days with what was called pleuro-pneumonia. 'This indicated that the pleura, ulcerated or softened by inflammation, was ruptured by the effort of straining, allowing air to escape into the pleural cavity. It is quite possible that the patient's memory was not accurate as to the number of days which elapsed between the onset of the inflammation and the appearance of dyspnœa. The long persistence of the difficulty indicated that the orifice remained open, for, had it closed, the air in the pleural cavity must soon have been absorbed, allowing the lung to expand, and the heart to return to the left side. It is also evident that the orifice was valvular, allowing air enough to escape into the pleural sac during inspiration to keep up the tension, and preventing its return during expiration. This is the reason that amphoric respiration was not heard, there being no free communication between the lung and the pleural cavity. The lung remained collapsed about its root, allowing a very faint vesicular murmur to be heard at the level of the axilla.

No effusion was found at any time while the patient was under observation at the House of Mercy. Had this occurred, it would have been recognized at once by dulness at the base, changing its location with change of position, and by the presence of metallic tinkling. Whether or not effusion had occurred at any time during the previous two years cannot be positively determined. The physician who attended him in the initial attack discovered none, so the patient thinks, but another, now deceased, who subsequently treated him for a time, told him he had hydrothorax. It is possible to question the correctness of this diagnosis, for it sometimes happens that the intensely tympanitic resonance is mistaken for dulness, and this doubt is strengthened by the subsequent existence for three years of air without fluid. As the attack was evidently inflammatory at the outset, it is very remarkable if the pneumothorax was unaccompanied by effusion; but it is no less remarkable if effusion took place, and the fluid was subsequently absorbed, leaving the cavity full of air, but one or the other of these phenomena must have occurred.

Although hydro-pneumothorax is by no means uncummon, ninety per cent. of the cases resulting from tuberculous ulceration, simple pneumothorax is rare. Flint relates one case in his own experience, and refers to another reported by Gairdner. In both these cases there was a rupture of the pleura by the strain, without previous inflammation. In Flint's case, the strain was due to carrying a pack on the back, the patient being a peddler. Another case without effusion has been reported by Dr. F. C. Shattuck, resulting from a stab in the chest. This was reported at a meeting of the Boston Society for Medical Improvement, on December 8,1884 , on which occasion Dr. Lyman exhibited three cases of hydro-pneumothorax - one traumatic, the second phthisical, and the third of pleuritic origin.

The duration of this case is exceeded by one reported by Laennec, which persisted for six years, but, with this exception, exceeds any which I have been been able to find.

As regards the treatment of this case, there is but little to say, for it received very little. The medicines which were administered during the first few months he was in the hospital had no appreciable effect, and he afterwards took none but tonics. Every effort was made to improve his general health, and an exception to the rules of the house was made in his case by permitting him to remain for so long a time, in order that he might avoid work and exposure. The propriety of removing the effused air by paracentesis was considered at various times, but was not resorted to, because it was believed that removing the pressure from the lung would only tend to delay the healing of the orifice, and it was not thought worth while, for the sake of a very temporary relief, to postpone the recovery.

The position habitually assumed by the patient during the fifteen months prior to his recovery may have perhaps exerted some beneficial influence. As already mentioned, he occupied himself with acquiring the art of stenography, and, during these latter months, spent ten or twelve hours a day in writing. While so engaged, the left foot was placed upon a stool, which raised it about eight inches from the floor, and the left elbow rested on the left thigh. This position would tend to reduce the action of the respiratory muscles of the left chest to a minimum, and thereby prevent the forcible suction of air inte the pleural sac. 'This would keep the valve of the pleural orifice at rest, and give its edges an opportunity to unite. The position was assumed by the patient because he found it very comfortable and restful, and very likely it answered the same purpose to some extent, as strapping the affected side, or fixing it with a plaster bandage, both of which have been recommended in such cases, the latter by Dr. F. 'T. Roberts, who has employed it with much benefit at the Brompton Consumptive Hospital.

\section{REPORT OF PROGRESS IN ORTHOPEDIC SURGERY.}

BY E. H. BRADFORD, M.D., AND R. W. LOVET', M.D.

DIRECT SURGICAL INTERFERENCE IN CERVICAL CARIES. ${ }^{2}$

Podres, in a case of severe cervical caries occurring in a child eleven years old, with evidence of deep abscess, made an incision two inches long, parallel to the posterior border of the sterno-cleido mastoid through the skin and fascia. and using a blunt instrument followed up the brachial plexus: pus was found on the sixth and seventh cervical vertebra, which were superficially ulcerated. The abscess wall and diseased bone were curetted and iodoform rubbed in, an antiseptic dressing applied with an extension for the head.

1 Concluded from page $3 \pi 6$.

2 Concluded from page 376. 